\title{
Non-destructive analysis of surface integrity in turning and grinding operations
}

Vladislav Ochodek, MSc., Department of Mechanical Technology, Faculty of Mechanical Engineering, VŠB-Technical University of Ostrava, 17 listopadu 15,708 33 Ostrava-Poruba, Czech Republic. E-mail: vladislav.ochodek@vsb.cz prof. Dr. Miroslav Neslušan, MSc., Martin Rosípal, MSc., Michal Š́pek, MSc., Department of Machining and Manufacturing Engineering, Faculty of Mechanical Engineering, University of Žilina, Slovakia. E-mail: miroslav.neslusan@fstroj.uniza.sk, martin.rosipal@fstroj.uniza.sk, michal.sipek@fstroj.uniza.sk

This paper deals with application of Barkhausen noise for analysis of surface integrity in turning and grinding operation. The stress state is analyzed in relation to tool wear. The results of measurements show that the residual stresses are not homogenous on the machined surface. This homogeneity depends on tool wear and stability of cutting process. Application of non-destructive testing through the Barkhausen noise is more suitable for grinding operations. Application of Barkhausen noise testing for turning operation will require the next research.

Keywords: Barkhausen noise, residual stress, grinding, turning

\section{References}

[1] NESLUŠAN, M. a kol. Experimentálne metódy v trieskovom obrábaní, EDIS Žilina, 2007, ISBN 978 - 80 - 8070 $811-8,349 \mathrm{~s}$

[2] KARPUCHEWSKI, B. Introduction to micro magnetic techniques, ICBM1 report Hanover 2002

[3] ABUKU, S., CULliTy,R. D. A Magnetic Method for the Determination of residual Stress, Exp. Mech. 11,1971

[4] ALTPETER, I.,THEINER, W. , BECKER, R. Eigenspannungsmessung an stal deer Güte $22 \mathrm{NiMoCr} 37$ mit magnetischen und magnetoelastischen Prüfverfahren, 4th Intern.Conf.on NDE in Nuclear Industry, Lindau1981

[5] KinOShitA, M., MURAYAMA, T., HOSHINA, N., KOBAYASHI, A., SHIMIZU, R., IKUTA, T. The surface damaged layer study of Mn-Zn single crystal ferrites using magnetic domain observation, CIRP, 1/1976, pp. 449

[6] THEINER, W. ,HÖLLER, P. Magnetishe Verfahren zur Spannungsermittlug, HTM - Beiheft Carl Hanser Verlag 1982

[7] BRINKSMEIER, E., SCHNEIDER, E. Nondestructive Testing for Evaluating Surface Integrity, CIRP, 2/1984, pp.489-496

[8] THEINER, W.A., WILLEMS, H.H. Determination of Microstructural Parameters, Symposium on Nondestructive Methods for Material Property Determination, april 6-8, 1983 Hesrhey, Pennsylvania

[9] NESLUŠAN, M. Sústruženie kalených ocelí, Edis Žilina 2010, ISBN 978-80-554-104-1

[10] WANG, J.Y. ,LIU, C. R. The effect of Tool Flank Wear on the Heat Transfer, Thermal Damage and Cutting Mechanics in Finishing Hard Turning, CIRP Annals 48/1/1999, p. 53 - 56

[11] BRANDT, D. Randzonenbeeinflussung beim Hartdrehen, Dr.-Ing. Dissertation, Universität-Hannover, 1995

[12] POKORNÝ, P., VÁCLAV, Š. Klasifikácia spôsobov rezania klinom. In: Vedecké práce MtF STU v Bratislave so sídlom v Trnave. - ISSN 1336-1589. - č. 25 (2008), s. 121-126

[13] WOJTAS, A. Analysis of residual stresses after shot peening. Strojírenská technologie, 2006, roč.XI.,č.3, s.6-9. ISSN 1211-4162.

[14] HOLEŠOVSKÝ, F. Stanovení zbytkových napětí v povrchu po obrábění. Strojírenská technologie, 2006, roč.XI.,č.3, s.29-32. ISSN 1211-4162.

[15] ŠTEKLÁČ, D., STANČEKOVÁ, D., ĎURECH, L., CZÁN, A. Zvyškové napätia při obránaní ložiskových materiálov a materiálov leteckých motorov. Strojírenská technologie, 2006, roč.XI., č.3, s.21-25. ISSN 1211-4162.

Reviews:

Prof. František Holešovský, MSc., Ph.D. Prof. Imrich Lukovics, MSc., Ph.D. 